\title{
CHROMOSOMAL POLYMORPHISM AND SALIVARY GLAND CHROMOSOMES OF HYBRIDS BETWEEN STRAINS OF ANOPHELES SINENSIS (DIPTERA: CULICIDAE) ${ }^{1)}$
}

\author{
YUZURU OGUMA \\ Department of Medical Zoology, School of Medicine \\ St. Marianna University, Sugao, Kawasaki 213
}

Anophles sinensis (Wiedemann 1828) is a member of the A. hyrcanus species group and the most wide-spread species in this species group. The distribution of the $A$. sinensis ranges from Japan and Korea in north, southwards through China to the Malay Peninsula. In southeast Asia, Assam is the weatern limit; it is absent from the rest of India (Reid 1968).

It is the medically important vector of a filaria, Brugia malayi (Filariae), in China and Korea (Feng 1931; Kanda et al. 1975) and is the supposed vector of malaria in southeast Asia. The speciation and evolution within A. sinensis as well as A. hyrcanus species group is of interest to geneticists and taxonomists and genetical analysis of these problems is now requited.

In general, a considerable amount of genetic variability concealed in natural populations of outbreeding diploid organisms. Some local populations of A. sinensis, the Tomakomai and the Engaru strains, are different than other $A$. sinensis strains with respect to the external morphology and the frequencies of the clasper movement in the male mosquitoes during induced copulation (Kanda and Oguma 1976). About 23\% of the Tomakomai and the Engaru strains of $A$. sinensis had $\mathrm{H} \mathrm{p}$ (humeral pale spots of wings), but that character could not be found in other $A$. sinensis strains. The mean frequencies of the clasper movements in the male adults of the Tomakomai and the Engaru strains of $A$. sinensis were 14.3 and 14.6, respectively, per one copulation induced and that of the original $A$. sinensis strain was 8.1. The difference in the frequencies of both was significant at the $1 \%$ level. Chromosomal polymorphism and the salivary gland chromosomes of hybrids in the strains have been studied in the present paper to see if the cytogenetic data might be correlated with the morphological observations and possible genetic divergence.

\section{MATERIALS AND METHODS}

For the study of chromosomal polymorphism in the strains of $A$. sinensis the materials were obtained at 11 localities, i.e. Koniya, Kanoya, Arao, Tokushima, Oka-

1) This study was supported by the grant from the Ministry of Education, the Government of Japan. 


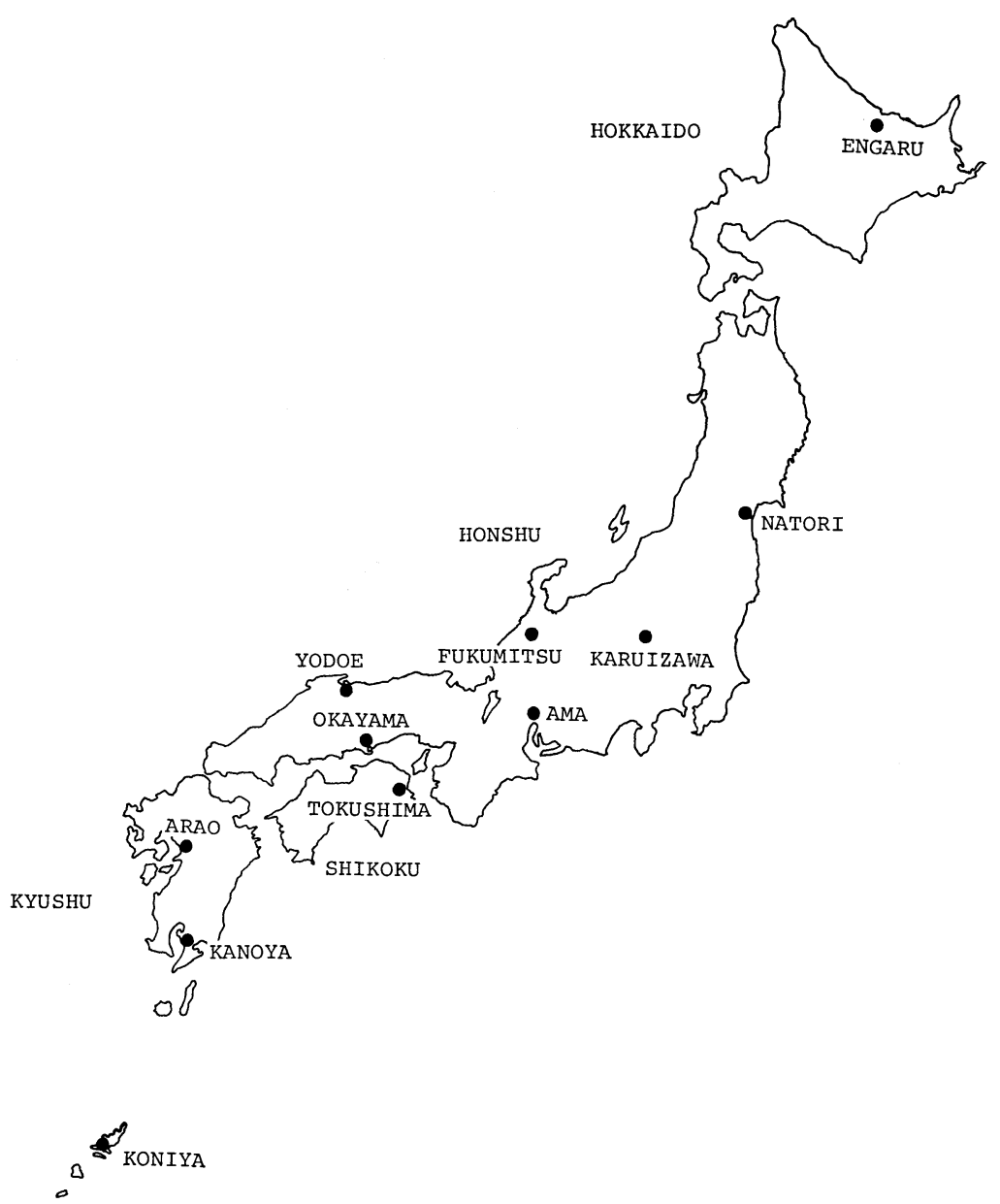

Fig. 1. Collecting localities of Anopheles sinensis.

yama, Yodoe, Ama, Fukumitsu, Karuizawa, Natori and Engaru, as given in Fig. 1. The methods of mosquito collection and rearing in the laboratory were the same as described in Oguma and Kanda (1976). About 50 females caught at each locality were allowed to lay eggs; these were reared to the fourth instar, then used for preparation of the salivary gland chromosomes. The techniques for preparation of the chromosome slides were similar to those described by French et al. (1962) and Kanda (1971). The number of the chromosomes observed was 60 for each of the arms except in the Koniya strain, in which it was 260; for a comparison of the chromosomal polymorphism, the salivary gland chromosomes of hybrids were made by induced copulation between any two of the Kanoya, Yakumo and Engaru strains.

\section{RESULTS}

Although two inversions, In2RA(12D-14B) and In3RA(28A-31B), had been found in the salivary gland chromosomes of this species (Kanda, unpublished), another simple 
Table 1. Chromosomal variability (\%) in Anopheles sinensis at 11 localities

\begin{tabular}{|c|c|c|c|c|c|}
\hline \multirow{2}{*}{ Locality } & \multirow{2}{*}{$\begin{array}{l}\text { No. of } \\
\text { chromosomes } \\
\text { observed }\end{array}$} & \multicolumn{2}{|c|}{ Chromosome 2R } & \multicolumn{2}{|c|}{ Chromosome 3R } \\
\hline & & ST* & In2RB & ST & In3RA \\
\hline Engaru & 60 & 1.65 & 98.35 & 95.00 & 5.00 \\
\hline Natori & 60 & 100 & 0 & 100 & 0 \\
\hline Karuizawa & 60 & 100 & 0 & 100 & 0 \\
\hline Fukumitsu & 60 & 100 & 0 & 98.35 & 1.65 \\
\hline Ama & 60 & 100 & 0 & 95.0 & 5.00 \\
\hline Yodoe & 60 & 100 & 0 & 100 & 0 \\
\hline Okayama & 60 & 100 & 0 & 100 & 0 \\
\hline Tokushima & 60 & 100 & 0 & 100 & 0 \\
\hline Arao & 60 & 100 & 0 & 100 & 0 \\
\hline Kanoya & 60 & 100 & 0 & 98.35 & 1.65 \\
\hline Koniya & 260 & 100 & 0 & 100 & 0 \\
\hline
\end{tabular}

* Standard arrangement

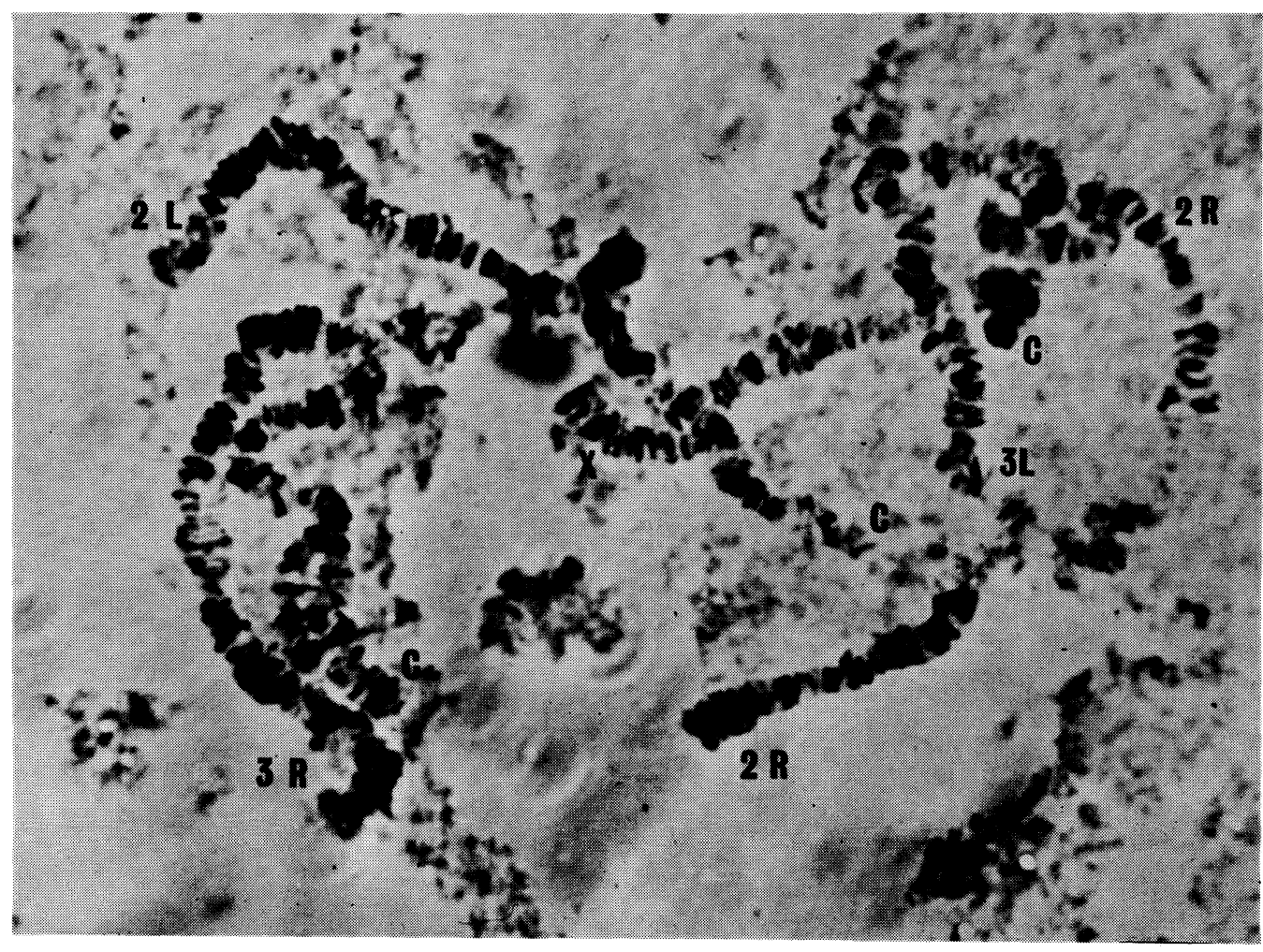

Fig. 2. Salivary chromosome complement of an $F_{1}$ between the Kanoya strain and the Yakumo strain of Anopheles sinensis. 


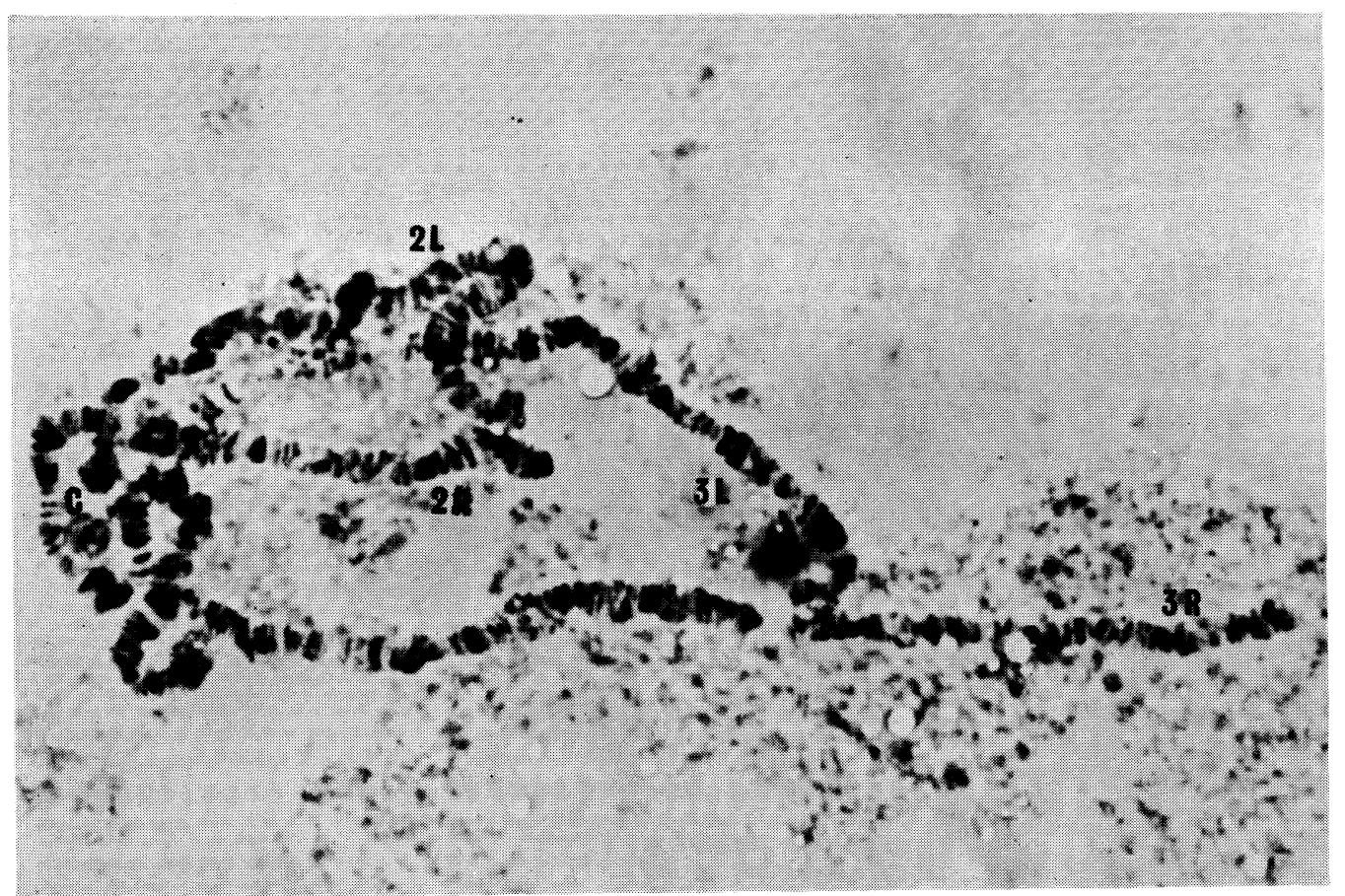

Fig. 3. Salivary chromosome complement of an $F_{1}$ between the Yakumo strain and the Kanoya strain of Anopheles sinensis.

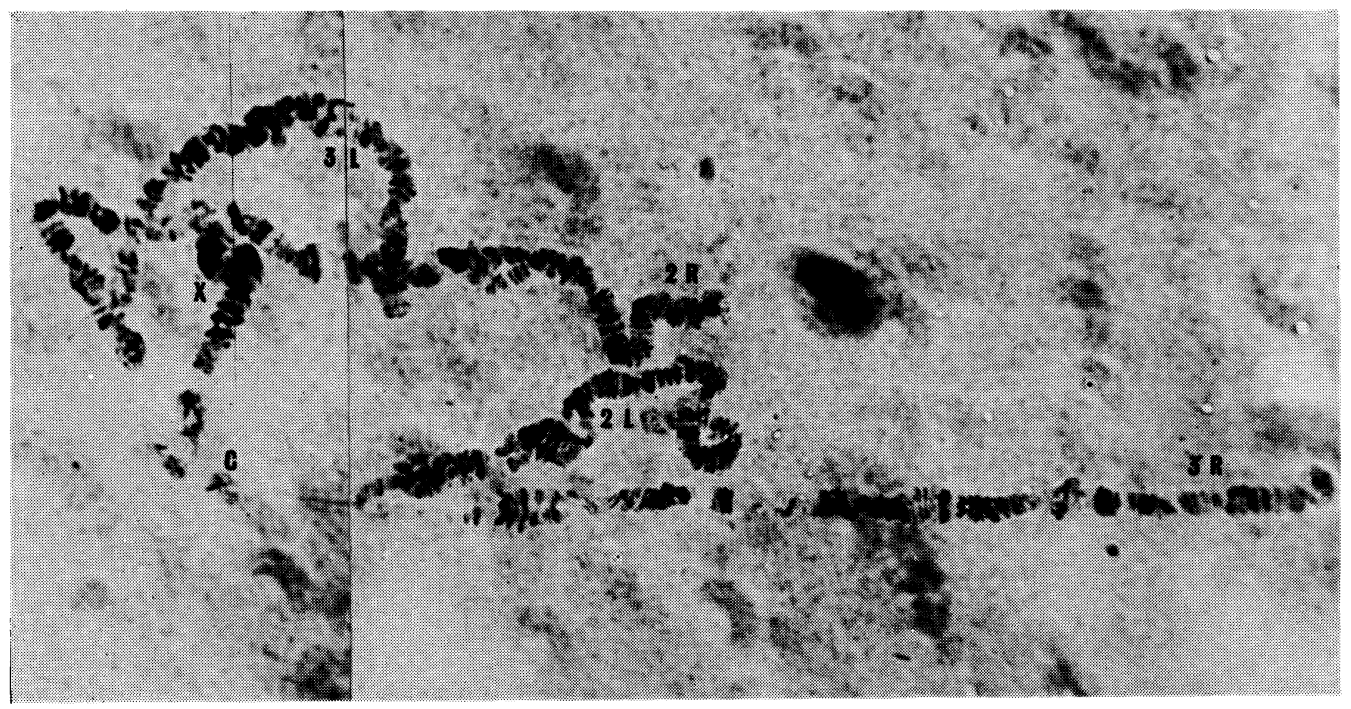

Fig. 4. Salivary chromosome of the Engaru strain of Anopheles sinensis. 


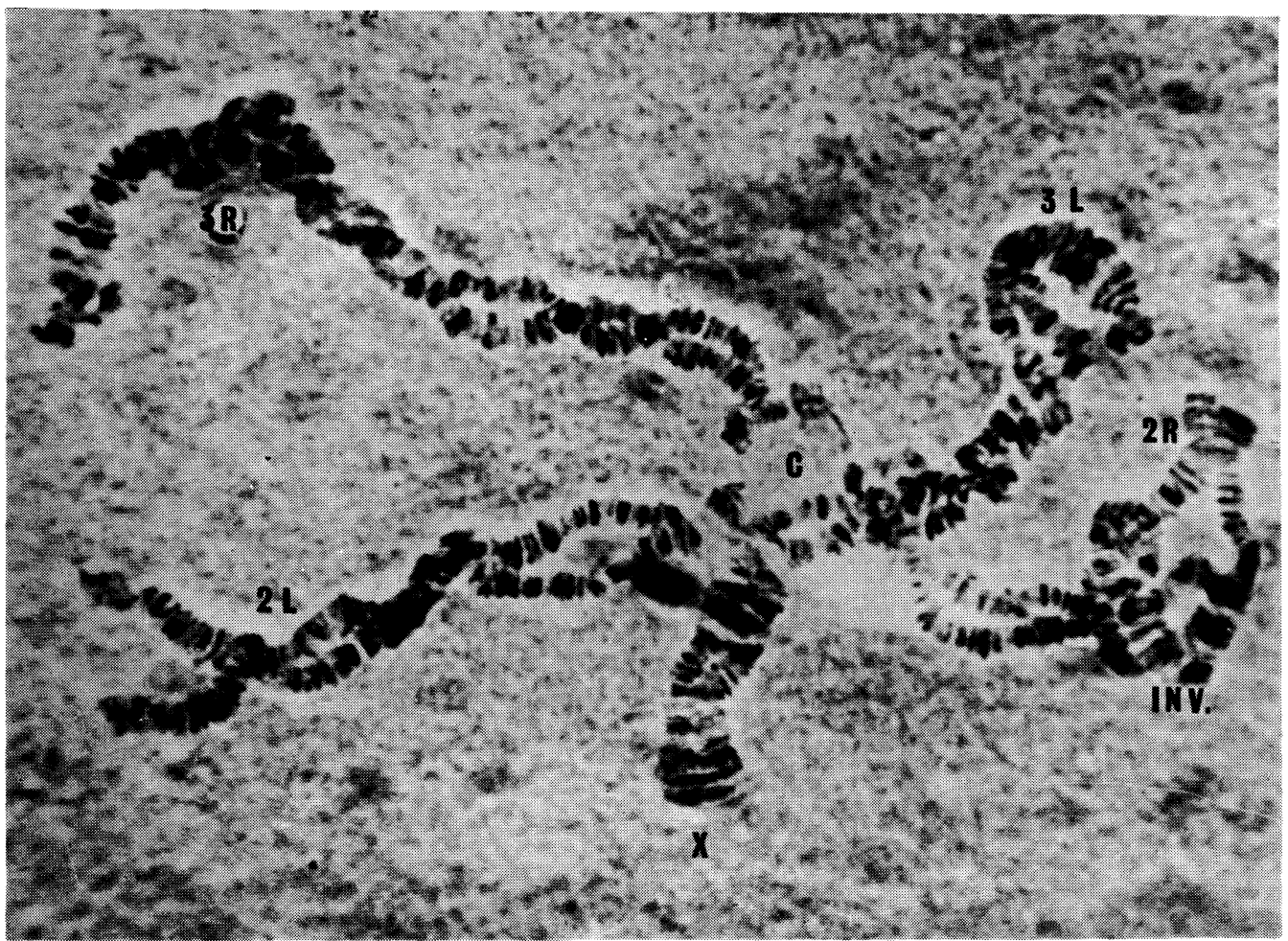

Fig. 5. Salivary chromosome complement of an $F_{1}$ between the Engaru strain and Yakumo strain of Anopheles sinensis; Incomplete synapsis along the whole lengths of the chromosome arms.

inversion, In2RB, was observed in the Engaru population, located at $9 \mathrm{~A}-11 \mathrm{D}$ on the right arm of the second chromosome. All the other regions of the chromosomal arms in the strains examined seemed to be the same as in the standard arrangement, and after all, the above mentioned three inversions have been found as polymorphisms of the salivary gland chromosomes in this species as summarized in Table 1. Inversion In2RA, which Kanda had found, could not be seen in any of the populations examined. Inversion In2RB was found only in the Engaru population and not in any other populations, whereas inversion In3RA was seen in the Engaru, Fukumitsu, Ama and Kanoya populations, although with very low frequencies at all of these localities.

Figs. 2 and 3 show the chromosome complements in the salivary glands of the hybrids between the female of the Kanoya and the male of the Yakumo strain, and of its reciprocal cross; in both the figures they are completely synaptic, although there is a small asynaptic region near the centromere in 3R. This small asynaptic region can usually be observed in $A$. sinensis and also even in the same strain, therefore this asynapsis is not due to hybridization between the two strains. The salivary gland chromosomes of the Engaru strain, which are shown in Fig. 4, show homologous pairing in the region of In2RB.

The hybrid chromosomes obtained from crosses between females of the Engaru and males of either the Yakumo or the Kanoya strains are presented in Figs. 5 and 


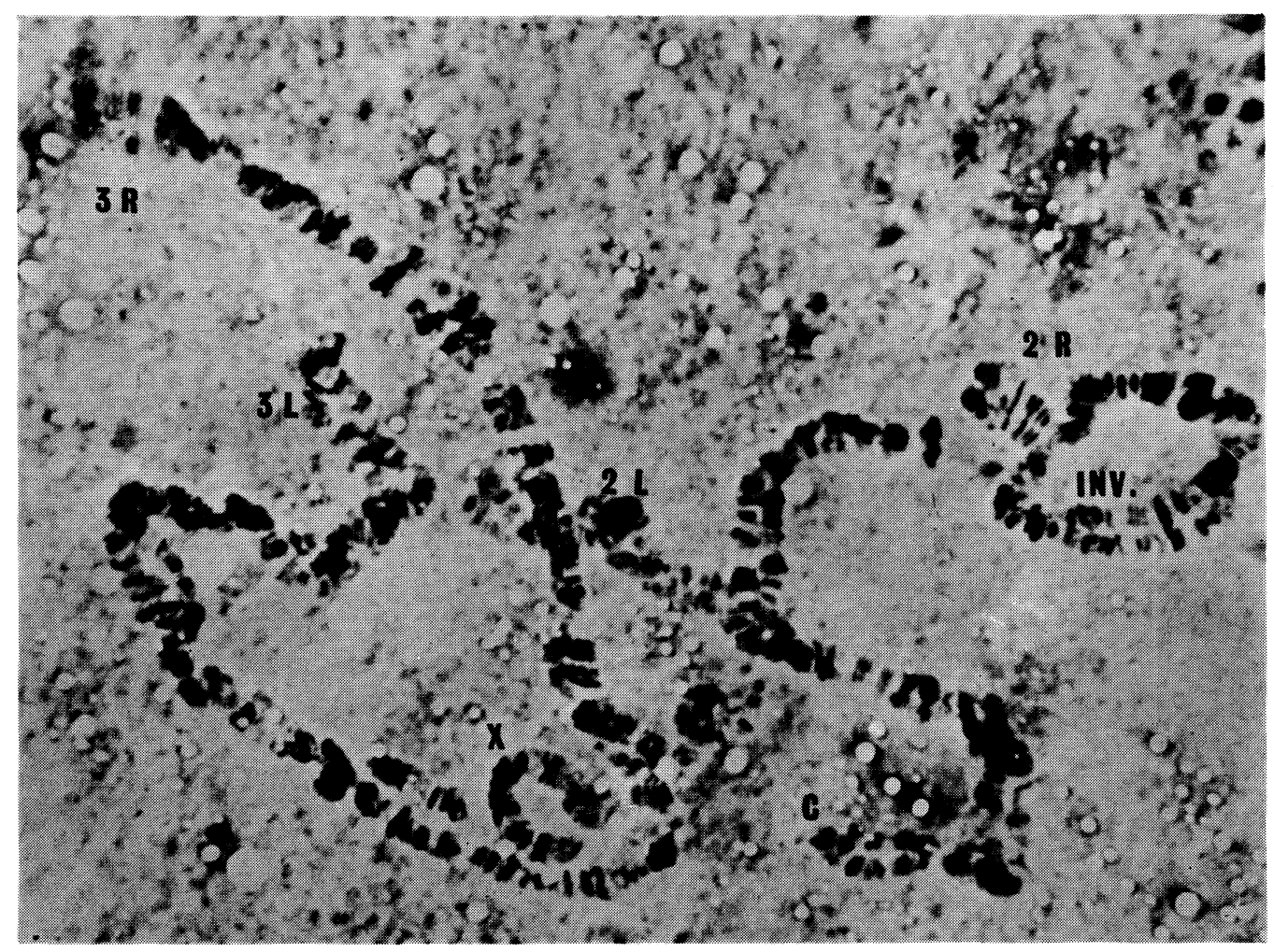

Fig. 6. Salivary chromosome complement of an $\mathrm{F}_{1}$ between the Engaru strain and Kanoya strain of Anopheles sinensis; Incomplete synapsis along the whole lengths of the chromosome arms.

6 ; in these figures incomplete synapsis is observed along the whole length of the chromosomal arms. In2RB is seen on the right arm of the second chromosome, while the X-chromosomes are not paired in Fig. 5, but are loosely paired in Fig. 6. When the Tomakomai strain was crossed with the Kanoya strain, the hybrid chromosomes showed complete synapsis in some crosses and incomplete synapsis in other crosses. Both complete and incomplete synapsis in this cross occurred with an almost equal frequency.

\section{DISCUSSION}

The investigation of inversion polymorphism has been useful for the study of evolution of species particularly in some families of Diptera. The most advanced and detailed investigations of this sort have been carried out in Drosophila. Anopheline mosquitoes are also favorable for the study of inversion polymorphism, for the salivary glands are good materials for the preparation of polytene chromosomes. The salivary gland chromosomes in A. maculipennis complex and in A. gambiae complex have been studied by Kitzmiller et al. (1967) and Davidson et al. (1967), respectively, who des- 
cribed many chromosomal inversions in these species, although with very little information on the quantitative aspects of the chromosomal polymorphism, whereas Coluzzi (1972) made a quantitative study of inversion polymorphism in 4 strains of A. stephensi.

Although a satisfactory quantity of materials for this study could not be acquired because of difficulties of preparation of the salivary gland chromosomes of $A$. sinensis, these data still can be useful for study of this species. Inversion In2RB exists in the Engaru population with a high frequency but was never found in any other populations thus far studied; this fact suggests that there is genetic divergence between the Engaru strain and strains of the original $A$. sinensis. Inversion In3RA is probably a common inversion, because it is found in populations from northern to southern parts of Japan with a low frequency. Inversion In2RA was found by Kanda (unpublished) from the Konosu population in Saitama Prefecture, near Tokyo and might exist in the populations with lower frequency than the In3RA. The salivary gland chromosomes of the $\mathrm{F}_{1}$ between the Kanoya and the Yakumo strains showed complete synapsis, suggesting that both the populations belong to the same species population. Incomplete synapsis of the salivary gland chromosomes was found in the $F_{1}$ between the Engaru and either the Kanoya or the Yakumo strain; the homologous chromosomes did not become completely parallel, and were loosely synaptic and twisted with each other in some parts.

According to Davidson et al. (1967), in the salivary gland chromosomes of the $F_{1}$ of A. gambiae $\mathrm{A}$ and $\mathrm{B}$, the $\mathrm{X}$-chromosomes and 7-11 of $2 \mathrm{R}$ are completely asynaptic; translocations are found between $2 \mathrm{~L}$ and $3 \mathrm{R}$; $3 \mathrm{~L}$ is completely synaptic along this arm. In the $A$. maculipennis complex, the salivary gland chromosomes of the $\mathrm{F}_{1}$ of $A$. freeborni $\times$ aztecus, freeborni $\times$ earle $i$, freeborni $\times$ occidentalis and earlei $\times$ occidentalis have been observed by Kitzmiller et al. (1967) to be completely asynaptic. The above species complex studied by Kitzmiller et al. can be identified by the banding patterns alone, on the other hand $A$. sinensis can not be distinguished from $A$. lesteri by the banding patterns of the salivary gland chromosomes, although the $F_{1}$ of both the species show completely asynaptic chromosomes (Kanda and Oguma, unpublished). Therefore the species which have homosequential banding patterns do not always show synapsis in their hybrid chromosomes. Based on the degree of synapsis it appears that there is more genetic divergence between the Engaru and Kanoya strains rather than between taxa $\mathrm{A}$ and $\mathrm{B}$ of $A$. gambiae, but less divergence than among A. maculipennis complex or between the A. sinensis s.l. and A. lesteri.

In the case of Drosophila, Drosophila bifasciata inhabits extensive areas in Europe and Asia including Japan, most commonly in cool and mountainous districts, while $D$. imaii has been collected only in Hokkaido. The salivary gland chromosomes in the $F_{1}$ of these species were observed to be incompletely synaptic in the second chromosome and the right arm of IV, but showed complete synapsis in the other arms (Moriwaki et al. 1967). They indicated that D. imaii was a new sibling species of D. bifasciata. By the same logic the Engaru strain can be considered to be a sibling species of the former $A$. sinensis. The salivary gland chromosomes of the $\mathrm{F}_{1}$ of the Tomakomai and Kanoya strains showed complete synapsis in some crosses and complete asynapsis in other crosses; this probably is because $A$. sinensis of the Engaru type and the original A. sinensis were mixed in the Tomakomai population. Further study is required to 
obtain the answer to this question.

\section{SUMMARY}

A new kind of inversion, named In2RB, has been found in the Engaru population of Anopheles sinensis. This is a simple inversion located at 9A-11D on the right arm of the second chromosome. Inversion polymorphism was investigated on In2RA, In2RB and In3RA in material from 11 localities. In2RA was not observed at all the localities and In2RB was found only in the Engaru population. In3RA was observed in four strains with low frequency. The hybrid chromosomes obtained from crosses between the Engaru strain and either the Kanoya or the Yakumo strains showed incomplete synapsis along the whole lengths of the chromosome arms.

\section{ACKNOWLEDGMENT}

The author wishes to express his thanks to Professor T. Kanda, St. Marianna University School of Medicine, for his suggestions which provided the initial stimulus for this investigation as well as for constant guidance in the course of the work. Thanks are also due to Emeritus Professor D. Moriwaki, Tokyo Metropolitan University, and Professor M. Sasa, National Institute for Environment Studies, for their advice and encouragement. $\mathrm{He}$ is indebted to Professors S. Ohba and O. Kitagawa, Tokyo Metropolitan University, and Dr. T. Tadano of the department, for their valuable suggestions and criticisms and to Emeritus Professor J. B. Kitzmiller, University of Illinois, for his constructive suggestions and reading the manuscript. Much valuable assistance in the laboratory was given by Miss K. Kuramochi.

\section{LITERATURE CITED}

Coluzzi, M., 1972 Inversion polymorphism and adult emergence in Anopheles stephensi. Science 176: $59-60$.

Davidson, M., H. E. Paterson, M. Coluzzi, G. F. Mason, and D. W. Micks, 1967 The Anopheles gambiae complex. In "Genetics of Insect Vectors of Disease" (J. W. Wright and R. Pal, ed.) pp. 211-260. Elsevier, Amsterdam.

French, W. L., R. H. Baker, and J. B. Kitzmiller, 1962 Preparation of mosquito chromosomes. Mosquito News 22: 377-383.

Kanda, T., 1971 On a observation method on salivary gland chromosomes in mosquitoes. Jap. J. Sanit. Zool. 20: 116.

Kanda, T., and Y. Oguma, 1976 Morphological variations of Anopheles sinensis Wiedemann, 1828 and A. lesteri Baisas and $\mathrm{Hu}, 1936$ and frequency of clasper movements of the males of several Anopheles species during induced copulation. Jap. J. Sanit. Zool. (In press)

Kitzmiller, J. B., G. Frizzi, and R. H. Baker, 1967 Evolution and speciation within the maculipennis complex of the genus Anopheles. In "Genetics of Insect Vectors of Disease" (J. W. Wright and R. Pal, ed.) pp. 151-210. Elsevier, Amsterdam.

Moriwaki, D., O. Kitagawa, and T. Okada, 1967 Drosophila imaii, a new sibling species related to Drosophila bifasciata. Evolution 21: 109-119.

Oguma, Y., and T. Kanda, 1976 Laboratory colonization of Anopheles sinensis Wiedemann, 1828. Jap. J. Sanit. Zool. (In press) 